\title{
TERAPIA INTRAVENOSA EM IDOSOS HOSPITALIZADOS: AVALIAÇÃO DE CUIDADOS
}

\author{
Ana Cláudia Roman Rós ${ }^{1}$, Daniela Ramos Oliveira ${ }^{2}$, Raquel Debon ${ }^{1}$, Maira Scaratti ${ }^{1}$
}

\begin{abstract}
RESUMO: Objetivou-se avaliar os cuidados de enfermagem na terapia intravenosa periférica em idosos internados ou em observação em um hospital-escola do sul do Brasil, nos meses de agosto e setembro de 2016. Pesquisa de natureza quantitativa prospectiva, avaliando as medidas de prevenção de infecção na terapia intravenosa, por meio de instrumento baseado nas recomendações da Agência Nacional de Vigilância Sanitária. Participaram do estudo 80 pacientes com idade média de 70,7 anos, sendo 51 (63,7\%) do sexo masculino. A identificação estava adequada em 15 (18,8\%) dos acessos venosos e o preenchimento correto dos rótulos não foi identificado em nenhuma solução avaliada. O curativo de fixação do cateter apresentava sujidade em $29(36,3 \%)$ casos e a incidência de flebite ocorreu em seis $(7,5 \%)$ pacientes. Concluiu-se que há inconformidades, que interferem na segurança do paciente hospitalizado, requerendo monitoramento da qualidade de assistência e educação permanente dos profissionais.
\end{abstract}

DESCRITORES: Idoso; Qualidade da assistência à saúde; Cuidados de enfermagem; Segurança do paciente; Infecção Hospitalar.

\section{INTRAVENOUS THERAPY IN HOSPITALIZED OLDER ADULTS: CARE EVALUATION}

\begin{abstract}
The aim of this study was to evaluate the nursing care related to peripheral intravenous therapy in older adults hospitalized or under observation in a teaching hospital in southern Brazil, between August and September 2016. This prospective quantitative study evaluated intravenous therapy infection prevention measures, by means of an instrument based on the recommendations of the Brazilian Health Regulatory Agency. The study included 80 patients with a mean age of 70.7 years, 51 (63.7\%) of whom were male The identification was adequate in $15(18.8 \%)$ of the venous accesses and the correct completion of the labels was not identified for any solution evaluated. The dressing for catheter fastening was unclean in $29(36.3 \%)$ cases and the incidence of phlebitis occurred in six $(7.5 \%)$ patients. It was concluded that there are nonconformities, which impact on the safety of the hospitalized patient, requiring monitoring of the quality of care and ongoing education of the professionals.
\end{abstract}

DESCRIPTORS: Older adult; Quality of healthcare; Nursing care; Patient safety; Hospital infection.

\section{TERAPIA INTRAVENOSA EN ANCIANOS QUE ESTÁN EN HOSPITAL: EVALUACIÓN DE CUIDADOS}

RESUMEN: Estudio cuyo objetivo fue evaluar los cuidados de enfermería en terapia intravenosa periférica de ancianos internados o en observación en un hospital-escuela de sur de Brasil, en los meses de agosto y septiembre de 2016. Investigación cuantitativa prospectiva que analizó las medidas de prevención de infección en la terapia intravenosa, por medio de instrumento que considera las recomendaciones de la Agencia Nacional de Vigilancia Sanitaria. Participaron del estudio 80 pacientes con edad media de 70,7 años, siendo $51(63,7 \%)$ del sexo masculino. La identificación estaba adecuada en 15 (18,8\%) de los accesos venosos y no se completó correctamente ninguno de los rótulos de la solución evaluada. El apósito de fijación del cateter presentaba suciedad en 29 (36,3\%) casos y hubo incidencia de flebitis en seis $(7,5 \%)$ pacientes. Se concluye que hay disconformidades que inlfluyen en la seguridad del paciente hospitalizado, lo que hace necesario monitorar la cualidad de asistencia y educación permanente de los profesionales. DESCRIPTORES: Anciano; Cualidad de la asistencia a la salud; Cuidados de enfermería; Seguridad del paciente; Infección Hospitalaria.

${ }^{1}$ Enfermeira. Residente em Saúde do Idoso e Atenção ao Câncer. Universidade de Passo Fundo. Passo Fundo, RS, Brasil. Enfermeira. Mestre em Envelhecimento Humano. Tutora do Programa de Residência Multiprofissional Integrada em Saúde do Idoso e Atenção ao Câncer da Universidade de Passo Fundo. Passo Fundo, RS, Brasil.

\author{
Autor Correspondente: \\ Ana Cláudia Roman Rós \\ Universidade de Passo Fundo \\ R. Paissandu 1932 - 99010-102 - Passo Fundo, RS, Brasil \\ E-mail: ana.claudiaros@gmail.com
}

Recebido: 28/12/2016 


\section{INTRODUÇÃO}

O envelhecimento é decorrente de processo multifatorial que engloba alterações em todos os sistemas do organismo e, desta forma, o cuidado ao paciente idoso difere dos pacientes adultos ${ }^{(1)}$. No ano de 2060, os idosos chegarão a 75,1 milhões de pessoas, representando $32,9 \%$ da população brasileira, ou seja, um em cada três brasileiros terá 60 anos ou mais ${ }^{(2)}$.

Segundo projeções, os idosos tendem a contribuir para a elevação do gasto assistencial, que passará de $27 \%$ do total em 2010 para $58 \%$ em $2050^{(3)}$. A internação passará a representar $60 \%$ destes gastos em $2050^{(3)}$. Esta população tende a consumir mais medicamentos, visto que a senescência está relacionada a alterações morfofisiológicas, maior suscetibilidade ao desenvolvimento de doenças crônicas e aparecimento de agravos. Por conseguinte, acabam utilizando grande parcela dos serviços de saúde ${ }^{(4)}$.

Os pacientes idosos têm alta morbidade e maiores chances de mortalidade em comparação aos indivíduos mais jovens. Quando associadas às infecções, as chances de mortalidade são mais evidentes ${ }^{(5)}$. Eleva-se o risco de desenvolver infecção relacionada à assistência à saúde, com destaque para corrente sanguínea ${ }^{(6)}$. A maioria das infecções dos acessos vasculares está relacionada ao cateter venoso central, entretanto, considera-se importante o acompanhamento de infecções relacionadas ao dispositivo periférico ${ }^{(7)}$.

A terapia intravenosa periférica é utilizada no tratamento de pacientes hospitalizados, é procedimento de rotina para os profissionais da enfermagem ${ }^{(8)}$. Envolve a colocação de dispositivo intravenoso em veia periférica e a instalação de soluções intravenosas conforme a necessidade de cada paciente, e tem como principais eventos adversos a flebite e o extravasamento de fluidos para o espaço extravascular, podendo causar prejuízos à rede venosa ${ }^{(9)}$.

Existe vulnerabilidade dos profissionais e risco de tornarem-se corresponsáveis por infecções relacionadas à assistência ${ }^{(10)}$. Isto provoca grandes desafios à saúde pública, que exige adequação dos serviços, disponibilidade de estrutura física, tecnologias específicas e profissionais capacitados ${ }^{(11-12)}$.

Com o progresso no desenvolvimento de tratamentos e drogas, a terapia intravenosa periférica vem se tornando cada vez mais complexa, aumentando os riscos de complicações para os pacientes ${ }^{(13)}$. Assim, conhecê-las, buscar elementos sobre práticas seguras, estar atento aos seus sinais e sintomas para preveni-los e tratá-los, devem ser prioridade da enfermagem.

Há déficit na literatura nacional e internacional sobre as particularidades do cuidado ao idoso em terapia intravenosa periférica e isso afeta diretamente a qualidade e a segurança da assistência de enfermagem a essa população, já que as diretrizes específicas não são corroboradas por estudos experimentais, clínicos ou epidemiológicos ${ }^{(14)}$.

Desta forma, este estudo objetiva avaliar os cuidados de enfermagem na terapia intravenosa periférica nos pacientes idosos internados ou em observação em um hospital- escola do sul do Brasil.

\section{MÉTODO}

Trata-se de uma pesquisa quantitativa prospectiva, com pacientes idosos, internados ou em observação em três unidades de enfermagem de um hospital-escola do sul do Brasil, sendo elas: Urgência e Emergência, Estudo Hemodinâmico e Internação Clínico Cirúrgico. A escolha dessas unidades deveu-se ao fato de atenderem, em sua maioria, idosos.

O hospital, campo de coleta de dados, tem caráter filantrópico, possui 631 leitos de internação e atende alta complexidade, sendo referência para os demais municípios da região.

Foram incluídos no estudo pacientes com 60 anos ou mais, em uso de terapia intravenosa periférica contínua ou intermitente por mais de 96 horas. Foram excluídos pacientes sedados e com alterações neurológicas.

A coleta de dados ocorreu nos meses de agosto e setembro de 2016, sendo aplicado instrumento elaborado a partir das recomendações da Agência Nacional de Vigilância Sanitária, do caderno Medidas 
de Prevenção de Infecção Relacionada à Assistência à Saúde ${ }^{(15)}$. A pesquisa ocorreu em um momento, com duração em média de 15 a 20 minutos.

Os itens avaliados pelas pesquisadoras foram: presença de material estéril utilizado para fixação/ curativo do acesso venoso periférico (AVP); curativo de fixação do AVP identificado com data, horário e rubrica do funcionário; presença de sujidade no curativo do AVP; registro em prontuário quando não realizada a troca rotineira do AVP a cada 96 horas; validade de equipos (96 horas) e soluções (24 horas) por meio de rótulos; identificação dos rótulos das soluções; presença de sinais de flebite (rubor, calor, dor e edema) no momento da avaliação.

Alguns itens foram questionados aos pacientes devido à impossibilidade de avaliação durante a aplicação do instrumento, as perguntas realizadas foram claras e com linguagem simples e em alguns casos realizaram-se demonstrações. As questões foram: qual instrumento utilizado para remoção dos pelos antes da punção do AVP; durante o banho ocorria a proteção do AVP com plástico; havia exame diário, através de palpação, do AVP; realização da desinfecção do dispositivo valvulado, com algodão e álcool, antes de conectar seringa ou soluções.

Os dados foram digitados em planilha eletrônica do programa Microsoft Office Exce $^{\circledR} 2010$ e analisados em software de estatística no programa Statistical Package for the Social Sciences ${ }^{\circledR}$ (SPSS) versão 22.0. Para variáveis quantitativas, calcularam-se as medidas de tendência central e dispersão. Os dados estão com frequências absolutas e relativas simples.

A pesquisa realizou-se após aprovação da Comissão de Pesquisa e Pós- Graduação do hospital onde ocorreu a coleta de dados e da aprovação do Comitê de Ética em Pesquisa da Universidade de Passo Fundo, no parecer $n^{\circ} 1.647 .147$.

Este estudo atende à Resolução 466/12 do Conselho Nacional de Saúde ${ }^{(16)}$; contempla o Código de Ética dos Profissionais de Enfermagem; atende aos aspectos éticos de consentimento do hospital; sigilo, anonimato e respeito aos valores dos participantes da pesquisa.

\section{RESULTADOS}

Participaram do estudo 80 pacientes com idade média de 70,7 $\pm 7,8$ anos, sendo 51 (63,7\%) do sexo masculino. Quanto à unidade, 26 (32,5\%) estavam na Urgência/Emergência, 26 (32,5\%) em Unidade de Internação Clínico Cirúrgico e 28 (35\%) no Estudo Hemodinâmico.

Tabela 1 - Medidas de prevenção de infecção relacionada à assistência à saúde na terapia intravenosa periférica. Passo Fundo, RS, Brasil, 2016 (continua)

\begin{tabular}{|c|c|c|c|c|c|c|c|}
\hline \multirow[t]{3}{*}{ ITENS AVALIADOS } & \multicolumn{6}{|c|}{ SETORES AVALIADOS } & \multirow{3}{*}{$\begin{array}{c}\begin{array}{c}\text { Total } \\
(\mathbf{n}=80) \\
\mathbf{n}(\%)\end{array} \\
\text { Sim }\end{array}$} \\
\hline & \multicolumn{2}{|c|}{$\begin{array}{c}\text { Urgência e } \\
\text { Emergência } \\
\begin{array}{c}n=26 \\
n(\%)\end{array}\end{array}$} & \multicolumn{2}{|c|}{$\begin{array}{l}\text { Unidade de } \\
\text { Internação } \\
\begin{array}{c}n=26 \\
n(\%)\end{array}\end{array}$} & \multicolumn{2}{|c|}{$\begin{array}{c}\text { Estudo } \\
\text { Hemodinâmico } \\
\begin{array}{c}n=28 \\
n(\%)\end{array}\end{array}$} & \\
\hline & Sim & Não & Sim & Não & Sim & Não & \\
\hline Identificação do curativo & $3(11,5)$ & $23(88,5)$ & $8(30,8)$ & $18(69,2)$ & $4(14,3)$ & $24(85,7)$ & $15(18,8)$ \\
\hline Avaliação diária do sítio de inserção & $23(88,5)$ & $3(11,5)$ & $21(80,8)$ & $5(19,2)$ & $16(57,1)$ & $12(42,9)$ & $60(75)$ \\
\hline Proteção do cateter durante o banho & $2(9,5)$ & $19(90,5)$ & $2(8,3)$ & $22(91,7)$ & $6(15)$ & $18(75)$ & $\begin{array}{c}10(14,5) \\
*\end{array}$ \\
\hline Desinfecção do dispositivo valvulado & $7(70)$ & $3(30)$ & $3(30)$ & $7(70)$ & - & - & $\begin{array}{c}10(50) \\
* *\end{array}$ \\
\hline Equipo dentro do prazo de validade & $10(62,5)$ & $6(37,5)$ & $21(87,5)$ & $3(12,5)$ & $10(100)$ & - & $\begin{array}{c}41(82) \\
* * *\end{array}$ \\
\hline $\begin{array}{l}\text { Solução contínua dentro do prazo de } \\
\text { validade }\end{array}$ & $10(71,4)$ & $4(28,6)$ & 17 (85) & $3(15)$ & $9(90)$ & $1(10)$ & $\begin{array}{l}36(81,9) \\
* * * *\end{array}$ \\
\hline
\end{tabular}


* Pacientes que realizavam banho de aspersão ( $n=69)$;

** Pacientes com dispositivo valvulado $(\mathrm{n}=20)$;

*** Pacientes que possuíam equipo de solução contínua ou intermitente $(n=50)$;

**** Pacientes em uso de solução contínua $(\mathrm{n}=44)$.

Foram avaliados 50 rótulos de soluções contínuas e intermitentes e $100 \%$ não estavam devidamente preenchidos com nome do paciente, leito, nome da solução, quantidade a ser infundida, data de instalação, horário e rubrica do profissional. Os demais pacientes estavam com o AVP salinizado, utilizado apenas no horário de administração de medicamentos.

Em dez pacientes (8\%) houve a remoção dos pelos para inserção do dispositivo venoso, destes, dois (20\%) informaram o uso de tricótomo elétrico, conforme recomendado. Em 17 pacientes (23\%) não foi realizada a troca do AVP após 96 horas, como é recomendado rotineiramente e estes não possuíam registro no prontuário sobre as condições e permeabilidade daquele dispositivo.

Para a fixação do cateter, não foram utilizadas coberturas estéreis na instituição em estudo, sendo esta uma recomendação. A presença de sujidade na fixação foi observada em $29(36,3 \%)$ pacientes. Quando avaliado por unidade de enfermagem, dos 26 pacientes da Urgência/Emergência, 13 (50\%) apresentavam o curativo com sujidade; já na Unidade de Internação, dos 26 pacientes avaliados, 10 $(38,5 \%)$ estavam sujos; em contrapartida, a unidade de Estudo Hemodinâmico foi a que apresentou a menor incidência de sujidade nas coberturas, sendo seis $(21,4 \%)$.

A ocorrência de evento adverso relacionado à terapia intravenosa periférica (flebite/extravasamento de fluídos) ocorreu em seis pacientes $(7,5 \%)$, sendo a incidência semelhante nos setores analisados (dois em cada setor).

\section{- DISCUSSÃO}

Estudos nacionais publicados sobre a adesão às medidas de prevenção de infecção de corrente sanguínea, relacionadas à terapia intravenosa periférica, envolvendo somente a população idosa, não foram encontrados, sendo estes mais voltados à população adulta ou pediátrica.

Neste estudo houve predominância de pacientes do sexo masculino, semelhante a publicação que avaliou a qualidade da assistência de enfermagem na terapia intravenosa periférica e sua amostra contou com $57,47 \%$ de homens ${ }^{(17)}$. A população masculina tem procurado mais o atendimento hospitalar, por condições de agravos a saúde, já que tende à menor adesão às medidas preventivas ${ }^{(18)}$.

No quesito identificação adequada do acesso vascular, a conformidade quanto ao preenchimento foi baixa, apenas 15 (18,8\%) estavam adequados. Em contrapartida, segundo estudo realizado em um hospital localizado no interior de São Paulo, das 720 observações 79,2\% dos acessos venosos periféricos estavam com identificação correta ${ }^{(19)}$.

Neste estudo, 60 (75\%) paciente relataram a avaliação diária do acesso venoso periférico (AVP) por profissional de enfermagem. A inspeção diária do acesso venoso periférico visa avaliar as condições do cateter, bem como a presença de sinais flogísticos sugestivos de infecção, sendo cuidado primordial de enfermagem ${ }^{(15)}$.

Quando questionados sobre o cuidado com o acesso durante o banho, apenas 10 (14,5\%) pacientes tiveram seus dispositivos protegidos com cobertura impermeável. A cobertura impermeável durante o banho e sua troca sempre que estiver sujo ou molhado ou com integridade comprometida são recomendadas ${ }^{(20)}$.

A desinfecção do disposto valvulado ocorreu em 10 (50\%) pacientes que utilizavam este tipo de conexão. Recomenda-se o uso de solução alcoólica e a realização de fricção no mínimo três vezes, antes de acessar o dispositivo. Também é necessário o monitoramento da incidência de infecção, após a introdução de dispositivos valvulados ${ }^{(15)}$.

A porcentagem de equipos e soluções dentro do prazo de validade conforme a Tabela 1 chegou a 
$82 \%$. Nos casos em que os pacientes apresentavam equipos e soluções fora do prazo de validade, as pesquisadoras comunicaram o enfermeiro gestor para que providenciasse as trocas. Estudo realizado em dois hospitais diferentes avaliou cinco indicadores de qualidade de enfermagem relacionados à terapia intravenosa periférica, dentre os resultados apresentou-se segura apenas para os indicadores identificação e validade dos equipos e frascos de soluções ${ }^{(21)}$.

Por outro lado, no estudo presente, a identificação correta dos rótulos das soluções foi nula, isto pode estar relacionado ao fato do desconhecimento de sua importância por parte da equipe de enfermagem, bem como à sobrecarga de trabalho. Os profissionais responsáveis pelo cuidado necessitam de educação permanente acerca das recomendações das medidas para prevenção de infecção da corrente sanguínea, e desta forma contribuirão para a segurança do paciente.

Podemos constatar que a tricotomia não foi realizada corretamente nas unidades avaliadas, ou seja, não ocorreu a remoção dos pelos com o dispositivo adequado. Isto pode se relacionar ao fato de que nem todas as unidades possuíam tricótomo elétrico. O uso deste dispositivo é fortemente recomendado pelo Centers for Disease Control and Prevention (CDC) ${ }^{(20)}$.

Neste estudo, em 17 (21,3\%) pacientes não foi realizada a troca do cateter venoso periférico após 96 horas como é rotineiramente recomendado, e estes não possuíam registro no prontuário sobre as condições e permeabilidade do dispositivo. A Anvisa recomenda que, nos casos em que não houve a troca do cateter, deve-se registrar as condições e permeabilidade do mesmo no prontuário do paciente ${ }^{(15)}$. No Brasil, a troca de cateteres tem sido empiricamente indicada pelas Comissões de Controle de Infecção Hospitalar de cada instituição(17).

É recomendada a troca rotineira do cateter venoso periférico a cada 72-96 horas em adultos e idosos, porém, quando clinicamente indicado, ainda é uma questão não resolvida, sugerindo que mais estudos são necessários, exceto para área pediátrica ${ }^{(20)}$. Conforme as recomendações da Anvisa, em pacientes neonatais e pediátricos não devem ser trocados rotineiramente os AVP e devem permanecer até completar a terapia intravenosa, a menos que indicado clinicamente ${ }^{(15)}$.

Alguns autores indicam um período três semanas sem complicações para utilização de cateter venoso periférico, pois troca rotineira causa muitos procedimentos invasivos desnecessários. Salientam que a política de troca rotineira de cateter venoso periférico tem sido aplicada por quatro décadas, tempo durante o qual o material dos cateteres evoluiu quanto à qualidade e durabilidade ${ }^{(22)}$.

Na instituição avaliada, não é preconizado o uso de cobertura estéril para fixação do cateter venoso periférico. É extremamente importante a adequada antissepsia da pele antes da punção venosa, assim como a fixação do cateter com adesivo estéril, preferencialmente transparente e de modo que possibilite a sua estabilidade ${ }^{(9)}$. A utilização de curativo estéril é fortemente recomendada ${ }^{(20)}$. A Anvisa também preconiza que o material que entra em contato com o sítio de inserção do cateter deve ser estéril, porém não sinaliza a categoria de recomendação(15).

Outra questão relevante foi a presença de sujidade no curativo de fixação do cateter, sendo identificada em 29 (36,3\%) dos pacientes. Desta forma, levanta-se questionamento acerca de como os profissionais avaliam as coberturas de fixação, pelo risco de infecções e agravos, bem como as orientações que o paciente recebe acerca dos cuidados com este dispositivo.

A incidência de eventos adversos (flebite e extravasamento) neste estudo ocorreu em seis (7,5\%) pacientes, não havendo predominância em uma unidade específica, porém, esta proporção é superior aos 5\% considerados como padrão aceito nas recomendações da Infusion Nurses Society ${ }^{(23)}$. Alguns autores constataram incidência de flebite de $31,1 \%$, estes descrevem que as instituições pesquisadas não adotavam o uso de curativos estéreis e sugerem isto como fator relacionado à alta proporção de flebite $^{(24)}$.

Os cuidados na manutenção do acesso, o controle das infusões, a prevenção de complicações e a monitorização constante estão entre as principais ações para a promoção da eficácia e segurança na terapia intravenosa ${ }^{(25)}$.

Os profissionais buscam o melhor para os seus pacientes. Porém, em decorrência da prestação de serviço estar ligada às complexas interações entre pessoas, instalações, equipamentos e medicamentos, 
a chance de o erro acontecer é elevada ${ }^{(26)}$. Outra questão importante é qual a compreensão que os profissionais têm da pessoa idosa e como isto interfere na maneira como é realizada assistência de enfermagem ${ }^{(27)}$.

Não existe um diagnóstico amplo sobre os problemas relacionados à terapia intravenosa periférica em pacientes idosos. Isto demonstra a necessidade de avaliação de indicadores de qualidade, bem como de educação permanente dos profissionais de saúde para reforçarem a cultura de segurança nos hospitais.

\section{CONSIDERAÇÕES FINAIS}

Ao analisar os itens de práticas seguras no cuidado com a terapia intravenosa periférica, identificaramse não conformidades, e estas interferem diretamente na segurança do paciente hospitalizado. A terapia intravenosa periférica é amplamente utilizada nos hospitais, mas pouco discutida em pacientes idosos. Este estudo teve algumas limitações, pois não avalia os profissionais de enfermagem e o que interfere na qualidade do cuidado prestado.

O monitoramento da qualidade da assistência se faz necessário no ambiente hospitalar, bem como refletir sobre a prática com base naquilo que é observado, elencar pontos que necessitam de melhorias, investir na educação permanente dos profissionais da saúde e reforçar a cultura de segurança do paciente.

\section{- REFERÊNCIAS}

1. Abrass IB, Kane RL, Ouslander JG, Resnick B. Fundamentos de geriatria clínica. 7a ed. Porto Alegre: Artmed; 2015.

2. Alves JED. Transição demográfica, transição da estrutura etária e envelhecimento. Revista Portal de Divulgação. [Internet] 2014;(40) [acesso em 20 jan 2016]. Disponível: http://www.portaldoenvelhecimento.com/revista-nova/ index.php/revistaportal/article/view/440/440.

3. Carneiro LAF, Campino ACC, Rodrigues FLCG, Santos GMM, Silva ARA. Instituto de Estudos de Saúde Suplementar (Org.). Envelhecimento populacional e os desafios para o sistema de saúde brasileiro. [Internet] São Paulo: IESS; 2013 [acesso em 10 fev 2016]. Disponível: http://documents.scribd.com.s3.amazonaws.com/ docs/1itre3bsjk3sac05.pdf.

4. da Silva EA, Macedo LC. Polifarmácia em idosos. Revista Saúde e Pesquisa. [Internet] 2013;6(3) [acesso em 03 mar 2016]. Disponível: http://periodicos.unicesumar.edu.br/index.php/saudpesq/article/view/2862.

5. Dimopoulos G, Koulenti D, Blot S, Sakr Y, Anzueto A, Spies C, et al. Critically ill elderly adults with infection: analysis of the extended prevalence of infection in intensive care study. J Am Geriatr Soc. [Internet] 2013;61(12) [acesso em 10 mar 2016]. Disponível: http://dx.doi.org/10.1111/jgs.12544.

6. de Sousa MAS, Lima TR, de Sousa AFL, Carvalho MM, Brito GMI, Camilotti A. Prevalência de infecção da corrente sanguínea em idosos internados em um Hospital Geral. Rev. Pre. Infec e Saúde. [Internet] 2015;1(3) [acesso em 15 mar 2016]. Disponível: http://www.ojs.ufpi.br/index.php/nupcis/article/view/4252.

7. Agência Nacional de Vigilância Sanitária (BR). Critérios Diagnósticos de Infecções Relacionadas à Assistência à Saúde. Brasília: Ministério da Saúde; 2013.

8. Batista OMA, Coelho SNOA, de Oliveira GM, Madeira AZA, Vieira CPB, dos Santos AMR. Fatores de risco para as complicações locais da terapia intravenosa periférica. Rev Enferm UFPI. [Internet] 2014;3(3) [acesso em 6 abr 2016]. Disponível: http://www.ojs.ufpi.br/index.php/reufpi/article/view/1540.

9. Harada MJCS, Pedreira MLG. Terapia intravenosa e infusões. São Caetano do Sul: Yendis; 2011.

10. Ataídes FS, Abrão FY, Costa CR, Silva MRR, Pimenta FC, Palos MAP, et al. Identificação de espécies de Candida em saliva de profissionais da saúde. Rev. Eletr. Enf. [Internet] 2010;12(3) [acesso em 10 abr 2016]. Disponível: http:// 
dx.doi.org/10.5216/ree.v12i3.6472.

11. Brito MCC, Freitas CASL, de Mesquita KO, Lima GK. Envelhecimento Populacional e os Desafios para a Saúde Pública: Análise da Produção Científica. Revista Kairós Gerontologia. [Internet] 2013;16(2) [acesso em 10 abr 2016]. Disponível: http://revistas.pucsp.br/index.php/kairos/article/view/18552/13738.

12. Ribeiro MG, Sancho LG, do Lago RF. Gastos com internação do idoso em serviços privados de terapia intensiva em três capitais da região sudeste: São Paulo, Rio de Janeiro e Belo Horizonte. Cad. saúde colet. [Internet] 2015;23(4) [acesso em 20 abr 2016]. Disponível: http://dx.doi.org/10.1590/1414-462X201500040096.

13. Modes PSSA, Gaíva MAM, Rosa MKO, Granjeiro CF. Cuidados de enfermagem nas complicações da punção venosa periférica em recém-nascidos. Rev. Rene. 2011;12(2):324-32.

14. Oliveira DFL, Azevedo RCS, Gaiva MAM. Guidelines for intravenous therapy in elderly: a bibliographic research. J. res.: fundam. care. online. [Internet] 2014;6(1) [acesso em 20 abr 2016]. Disponível: http://dx.doi. org/10.9789/2175-5361.2014v6n1p86.

15. Agência Nacional de Vigilância Sanitária (ANIVSA). Medidas de Prevenção de Infecção Relacionada à Assistência à Saúde. Brasília: ANVISA; 2013.

16. Ministério da Saúde (BR). Conselho Nacional de Saúde. Diretrizes e Normas Regulamentadoras de pesquisas envolvendo seres humanos. Brasília. Resolução nº 466, de 12 de dezembro de 2012. Brasília; 2012.

17. de Souza AEBR, Oliveira JLC, Dias DC, Nicola AL. Nursing care quality in peripheral intravenous therapy: analysis by indicators. Cogitare Enferm. 2014;19(3):478-84.

18. Cunha BSS, Nascimento AS, Sá SPC. Perfil clínico e sociodemográfico de internação de idosos na unidade de emergência de um hospital geral. Estud. interdiscipl. envelhec. 2014;19(1):189-200.

19. Caldana G, Gabriel CS, Bernardes A, de Pádua RX, Vituri DW, Rossaneis MA. Avaliação da qualidade de cuidados de enfermagem em hospital público. Semina: Ciências Biológicas e da Saúde. [Internet] 2013;34(2) [acesso em 25 abr 2016]. Disponível: http://dx.doi.org/10.5433/1679-0367.2013v34n2p187.

20. O'Grady NP, Alexander M, Burns LA, Dellinger EP, Garland J, Heard SO, et al. Guideline for the prevention of intravascular catheter-related bloodstream infections. [Internet] Atlanta: CDC; 2011 [acesso em 15 jan 2016 ]. Disponível: https://www.cdc.gov/hicpac/BSI/BSI-guidelines-2011.html.

21. Murassaki ACY, Versa GLGS, Bellucci Júnior JAB, Meireles VC, Vituri DM, Matsuda LM. Avaliação de cuidados na terapia intravenosa: desafio para a qualidade na enfermagem. Esc. Anna Nery. [Internet] 2013;17(1) [acesso em 02 fev 2016]. Disponível: http://dx.doi.org/10.1590/S1414-81452013000100002.

22. Rickard CM, Webster J, Wallis MC, Marsh N, McGrail MR, French V, et al. Routine versus clinically indicated replacement of peripheral intravenous catheters: a randomised controlled equivalence trial. Lancet. [Internet] 2012;380(9847) [acesso em 01 mai 2016]. Disponível: http://dx.doi.org/10.1016/S0140-6736(12)61082-4.

23. Infusion Nurses Society. Infusion nursing standards of practice. J Infus Nurs. 2006;29(Suppl 1):S1-92.

24. Enes SMS, Opitz SP, de Faro ARMC, Pedreira MLG. Phlebitis associated with peripheral intravenous catheters in adults admitted to hospital in the Western Brazilian Amazon. Rev. esc. enferm. USP. [Internet] 2016;50(2) [acesso em 04 mai 2016]. Disponível: http://dx.doi.org/10.1590/S0080-623420160000200012.

25. Cardoso JMRM, Rodrigues EC, Rodrigues BMRD, Pacheco STA, Faria JCO. Escolha de veias periféricas para terapia intravenosa em recém-nascidos pela equipe de enfermagem. Rev. Rene. 2011;12(2):365-73.

26. Wachter R. Compreendendo a Segurança do Paciente. $2^{\mathrm{a}}$ ed. Porto Alegre: AMGH; 2013.

27. Schimidth TCG, da Silva MJP. The perception and understanding of health professionals and undergraduate students regarding aging and the elderly. Rev. esc. enferm. USP. 2012;46(3):612-17. 